\title{
Cognitive Consequences of Social Isolation During COVID-19: side effects and treatments
}

\author{
Zahra Taherizadeh ${ }^{1}$, shayan rahmani $^{1}$, Fatemeh Alidadi ${ }^{1}$, and Khadijeh Esmaeilpour ${ }^{1}$ \\ ${ }^{1}$ Affiliation not available
}

February 23, 2021

\begin{abstract}
Objective: During the COVID-19 pandemic, quarantine and staying at home is advised. The social relationship between people has become deficient, and human social isolation (SI) has become the consequence of this situation. It was shown that SI has made changes in hippocampal neuroplasticity, which will lead to poor cognitive function and behavioral abnormalities. There is a connection between SI, learning, and memory impairments. In addition, anxiety-like behavior and increased aggressive mood in long-term isolation have been revealed during the COVID-19 outbreak. Methods: Term searches was done in Google Scholar, Scopus, ScienceDirect, Web of Science and PubMed databases as well as hand searching in key resource journals from 1979-2020. Results: Studies have shown that some drug administrations may positively affect or even prevent social isolation consequences in animal models. These drug treatments have included opioid drugs, anti-depressants, Antioxidants, and herbal medications. In addition to drug interventions, there are non-drug treatments that include an enriched environment, regular exercise, and music. Conclusion: This manuscript aims to review improved cognitive impairments induced by SI during COVID-19.
\end{abstract}

\section{Cognitive Consequences of Social Isolation During COVID-19: side effects and treatments}

Zahra Taheri Zadeh ${ }^{\text {a }}{ }^{1}$, Shayan Rahmani ${ }^{\text {a }}{ }^{2}$, Fatemeh Alidadi ${ }^{3}$, Khadijeh Esmaeilpour ${ }^{1 *}$

${ }^{1}$ Neuroscience Research Center, Institute of Neuropharmacology, Kerman University of Medical Sciences, Kerman, Iran

${ }^{2}$ Student Research Committee, Shahid Beheshti University of Medical Science, Tehran, Iran

${ }^{3}$ Department of Biology, Payam Noor University, Tehran, Iran

* Corresponding author at: Neuroscience Research Center, Institute of Neuropharmacology, Kerman University of Medical Sciences, Kerman, Iran

E-mail address: esmaeilpour@kmu.ac.ir

${ }^{a}$ Both authors contributed equally to this manuscript

\section{Abstract}

Objective: During the COVID-19 pandemic, quarantine and staying at home is advised. The social relationship between people has become deficient, and human social isolation (SI) has become the consequence of this situation. It was shown that SI has made changes in hippocampal neuroplasticity, which will lead to poor cognitive function and behavioral abnormalities. There is a connection between SI, learning, and memory impairments. In addition, anxiety-like behavior and increased aggressive mood in long-term isolation have been revealed during the COVID-19 outbreak.

Methods: Term searches was done in Google Scholar, Scopus, ScienceDirect, Web of Science and PubMed databases as well as hand searching in key resource journals from 1979-2020. 
Results: Studies have shown that some drug administrations may positively affect or even prevent social isolation consequences in animal models. These drug treatments have included opioid drugs, anti-depressants, Antioxidants, and herbal medications. In addition to drug interventions, there are non-drug treatments that include an enriched environment, regular exercise, and music.

Conclusion: This manuscript aims to review improved cognitive impairments induced by SI during COVID19.

Keywords: COVID-19, social isolation, learning and memory, anxiety, drug treatments, non-drug treatments

\section{Introduction}

Social interaction has a crucial role in human well-being, both mentally and physically (1). Human social isolation (SI) happens when the social relationship between individuals become deficient (2). Moreover, it mostly occurs when the number of individuals, who are members of social links, decrease, or the qualification of social relations, diminishes. During SI, people experience unpleasant situations mentally, emotionally, and spiritually $(3,4)$. Some conditions force individuals to leave human groups and reduce their social interactions, presence in the population, and group activities. Being single, getting a divorce and separation may also result in isolation $(2,5)$. Further, weak connections and lack of social support have shown to be significant risk factors of isolation, which result in loneliness, stress, and committing suicide $(6,7)$. Meanwhile, some infectious diseases like COVID-19, AIDS, and some physical disorders have shown to develop SI in humans $(8,9)$.

Adverse effects on cognition and behavior, decision-making, and pain perception are followed by social isolation $(10,11)$. SI has shown to change the immune system, glutamate system, and hormones $(12,13)$. Besides, cardiovascular disease, high blood pressure, stroke, and developmental neurodegenerative diseases have occurred during SI (14). Depression was also induced while motor dysfunction decreased during isolation (15-17). Some evidence demonstrated more oxidative stress and inflammation because of increased IL-1ß, cytokines, and brain macrophages during SI (18). Isolation and loneliness lead to a higher rate of morbidity, mortality, and it is strongly related to chronic disease with death in adults $(17,19)$.

Previous researches have shown there is a relation between SI and alternations in the hippocampus. According to studies, changes in hippocampal neuroplasticity will lead to poor cognitive function and behavioral abnormalities $(20,21)$. There is a connection between SI, learning, and memory impairments as well. Some studies have reported that environmental factors play a significant role in brain development and cognitive function in rodents, which directly affect learning and memory performance $(22,23)$. Results suggest that SI as the lack of presence of others or the few numbers of meaningful relationships is vastly associated with many aspects of memory and learning impairment $(24,25)$. Morris Water Maze's outcomes have also shown spatial learning and memory dysfunction during isolation $(26,27)$. Other studies have demonstrated inhibition of autophagy by the production of some factors (28), deficit spatial learning and memory, social recognition memory, reversal learning, and short-term memory during SI periods (29). Alternatively, some studies have shown that isolated environments did not affect spatial learning and memory, spatial reference memory, reversal learning, and short-term memory. Moreover, in some cases, results have suggested that memory and learning performance has improved due to social isolation $(28,30)$.

Animal research indicated depression, anxiety-like behavior, and increased aggressive mood in long-term isolation $(31,32)$. Moreover, the time spent in the open arms of the Elevated plus Maze has decreased as a consequence of higher anxiety (33). Chronic stress has taken place as a feature of anxiety and depression.

The human species depends on social behavior and social interaction. Being social helps humans survive and solve problems; while, weak social interaction negatively affects social memory and sociability during SI (34, $35)$. There has been a correlation between neuroendocrine disability and impairments in neurogenesis of the hippocampus and decline in BDNF expression (36).

The results have also revealed that SI has developed Alzheimer's disease, and it has exacerbated spatial learning impairment in aged mice (37). Motor activity is not affected according to open field outcomes; 
however, chronic stress during SI has shown to the deficit the motor function in rodent models of Parkinson's disease $(38,39)$.

\section{Drug treatments}

Studies have shown that some drug administrations may positively affect or even prevent social isolation consequences in animal models.

1. Opioid drugs: These are measured by pinning configuration and rats use them to defend nap access. According to previous play investigation, fighting became greater in junior rats during short-term social deprivation. Social grooming, as a behavioral factor by young and adult animals, is performed in rodents and primates. Both behavioral responses among primates, particularly humans, accounted for increasing isolation due to neurochemicals and opioid systems involvements (40,41, 42).

Naltrexone treatment by subcutaneous injection in a dose range of $0.03-1 \mathrm{mg} / \mathrm{kg}$ body weight was administered. It has decreased pinning duration, frequency, and grooming behavior dose-dependently $(41,43)$.

Apomorphine treatment by subcutaneous injection in 2 doses of 60 and $100 \mathrm{pg} / \mathrm{kg}$ has significantly reduced pinning duration and frequency in a dose-dependent manner. In addition, a $35 \mathrm{pg} / \mathrm{kg}$ dose of apomorphine decreased grooming behavior $(41,44)$.

$\beta-\varepsilon \nu \delta o \rho \pi \eta \nu$ treatment in a dose of $100 \mathrm{pg} / \mathrm{kg}$ has decreased the duration of pinning, but not for the time of grooming.

In conclusion, some opioid drugs have improved social activity by regulating the opioid receptors and neuronal systems $(41,45)$.

2. Oxytocin: Genetically, lower levels of oxytocin in some mice or hypersensitive receptors to some stressors caused mental illness and reduced social interaction (46). On the other hand, stressful experiences and accidents may result in overproduction of oxytocin in the central and peripheral nervous system. Longterm SI significantly led rodents to be immobile, less active, and depressed. The social environment has regulated oxytocin productions in specific regions in the CNS $(47,48)$.

In some rodents, administration of exogenous oxytocin for a long time has blocked weak social contact and behavioral impairments, such as depression during SI(49). However, oxytocin can perform as an antidepressant drug and decrease negative social interactions as well as encourage isolated ones to get over it (50). Other investigations have described how oxytocin developed stress response consequences of separation in female rodents (51-53).

Stress is often accompanied by isolation-induced alternations on neurochemicals; subsequently, depression and anxiety disorder. Previous studies have revealed the injection of oxytocin with inter-central amygdala procedure in mice resulted in improvement in depression and anxiety behavior (54). Many investigations have explained, amygdala activity has been regulated by oxytocin. Oxytocin can also perform as an anti-anxiety and anti-depressant drug to treat isolation-induced social stress after 5-weeks of social isolation. Longterm SI has decreased OXTR mRNA transcription and GABA level in mice and induced anxiety-related behavior and depression $(46,55)$. Oxytocin administration has attenuated depressive-like, anxiety-like, and destructive social behaviors. Anxiety measurement was performed with an open field test (OFT) and the elevated zero maze test (EZMT) in mice $(48,56)$.

3.Antidepressant drugs: According to previous investigations, decreased BDNF level and neuro-steroids in the hippocampus were induced by SI and depression in adults. Published data have shown antidepressant treatment like fluoxetine and fluvoxamine increased BDNF mRNA expression $(57,58)$. Neuro-steroids such as allopregnanolone reduced aggression and anxiety-like behaviors. The stable emotional mood in rodents has made them successful in improving social support and less isolation because of allopregnanolone performance on GABA neurotransmitters $(59,60)$. 
It has been revealed that a selective serotonin (5-HT) reuptake inhibitor such as Fluoxetine is able to alleviate isolation-induced depression behavior (61). Oral Fluoxetine consumption has improved the depletion of serotonin in the hippocampus; and, anxiety, depression, and social deficits have reversed during social isolation. Moreover, brain neurogenesis has improved, which plays a crucial role in emotional deficit development (62-64). With Fluoxetine treatment, as an anti-depressant, metabolic impairment were also reversed (65). Some documents have claimed that Fluoxetine that is attached to mitochondria can change anion channels voltage, and finally, alleviate depression $(64,66)$.

Clozapine has been known as an effective drug to improve the social deficit induced by SI. This research has shown alternation in corticostriatal ATP levels, anti-inflammatory cytokines, and neuroprotective ratio through isolation condition (67). The whole alternations were reversed by Clozapine, as an atypical antipsychotic, to improve SI detriments and particularly depressive behaviors (68). Anti-depressant properties of Clozapine have made it beneficial to prevent isolation-induced depression in rats (69). Molecular investigations have shown Clozapine has decreased TNF- $\alpha$, GPX, and glutamate-like receptor significantly; plus, less GLR activity of cyclooxygenase-2 (COX-2) and interleukin-1beta (IL-1 $\beta$ ) in the hippocampus were induced by Clozapine (70). Administration of Fluoxetine and Clozapine, as nonsteroidal anti-inflammatory drugs for three weeks in rats, prevented quanta decline in hippocampal parvalbumin-positive (PV+) cells $(68,71)$.

Increased GSH content and nuclear factor-kappa B (NF-kB) has led to reduced depressive-like and anxietylike behaviors in isolated rats (72); while Leponex ( $25 \mathrm{mg}$ of CLZ per tablet) was administrated for 21 days. More research has revealed low doses of Clozapine $(0.1,0.2$, and $0.4 \mathrm{mg} / \mathrm{kg}$ ) can exert anxiolytic properties in isolated rats and reduce anxiety behavior, stress, and depressive mood (68).

Besides, chronic administration of Fluoxetine is effective in treating SI-induced impairments of spatial learning and memory, cognition, neurogenesis, emotion-related, and depressive-like behaviors in rodents (65). Further, Clozapine has found to improve behavioral deficits and activate some regions in the brain, such as dHIPP and RSC, associated with memory, learning, and spatial orientation in socially isolated rats. Moreover, long-term administration (6-8 weeks) of Clozapine (5 or $10 \mathrm{mg} / \mathrm{kg}$ ) has shown to improve the reversal learning deficit in SI rats $(66,73,74)$.

Another investigation revealed that 5 to 10 days of consumption of antipsychotic drugs, like Ampakine and Aniracetam, have reversed the impairment of recognition memory in isolation-reared rats (75-77). Further, administration of Methylphenidate (1-10 mg/kg) and Caffeine $(0.5-1 \mathrm{mg} / \mathrm{kg})$, which are commonly used for attention deficit hyperactivity disorder (ADHD), have shown to be efficient for latent learning and spatial attention impairment (78). Moreover, a low dose of corticosterone increased the expression of the activityregulated cytoskeletal associated protein (Arc) and improved long-term memory in socially isolated rats. Other studies have shown that inhibiting receptors via antagonist drugs might reverse SI impairment in rodents (79-81).

Administration of 5-HT6 receptor antagonist drugs can potentially bring back learning, cognition, and recognition memory deficit by up-regulating glutamate and serotonin in cortical and hippocampal regions in SI-reared rats (82). Results suggeste that PRX-07034 and PRX-07037, as 5-HT6 antagonists, reverse the isolation rearing-induced memory deficit, while Ro 04-6790 diminishes the effect of isolation on reversal learning impairment $(75,83,84)$. In addition, Ro $\mathbf{4 3 6 8 5 5 4}$ has been able to reverse a scopolamine-induced impairment in emotional learning (85).

SI has shown to elevate Rac1 activity in hippocampal tissue, inducing social recognition memory (SRM) forgetting and long-term potentiation (LTP) decline in mice; According to this finding, Inhibiting of Rac1 Activity Blocked Progressed Decline of LTP and suppressed forgetting of SRM in isolated adult mice. However, Rac1 activity had no influence on short-term (15-min) memory in the socially isolated period (86).

Additionally, Results have revealed that in socially isolated mice, the excitatory presynaptic release of pyramidal neurons in the $\mathrm{mPFC}$ has attenuated, and metabotropic glutamate receptor $\mathbf{2 / 3}$ (mGluR2/3) antagonist, LY341495, played a crucial role in recovering working memory by building reasonably vast synaptic strength in the mPFC in SI-reared mice (87, 88). Further, a single treatment with LY341495 
improved isolated mice performance in the $\mathrm{Y}$ maze test but not in the novel object recognition test, while repeating the treatments were efficient for both tasks $(87,89)$. Conversely, mGluR2/3 agonist, LY379268 , has also improved recognition memory impairment in SI rats $(87,90)$. Earlier investigations have suggested that stress and anxiety-like behaviors have appeared due to a mediator called the Corticotropin-releasing factor $(\mathrm{CRF})$. CRF agonist injection has shown additional anxiety in the Elevated plus Maze and open field tests in rats. During isolation and social impairment, the CRF receptor has been increasingly activated in the DRN and this result in anxiety-like behaviors. Subsequently, researchers have administered CRF receptor antagonists, which decreased stress and anxiety $(91,92)$.

4. Antioxidants: Isolation-induced oxidation stress has led to many cognitive impairments, such as violence, aggression, and anxiety. Reactive Oxygen Species have been produced by oxidative stress and they caused variable damages to the brain structurally and physiologically (93). Researchers have decided to evaluate the administration of antioxidants in isolated mice to reduce social and behavior deficits like aggression for 14 days. Eventually, results have revealed vitamin $\mathrm{E}$ in high doses and $\mathrm{N}$-acetyl cysteine in low doses were effective to decline aggression in isolated mice. A low dose of vitamin $\mathrm{E}$ and $\mathrm{N}$-acetyl cysteine beta-carotene in high doses, were effective in reducing acute isolation-induced aggressive behaviors. However, ascorbic acid has exhibited a more dose-dependent behavior. Biochemistry procedures have evaluated antioxidant markers; while, molecular results have shown the level of catalase, superoxide dismutase enzymes, and glutathione. Data have suggested an increase in biomarkers among isolated mice treated with antioxidants. Researchers found that antioxidants consumption after 14 days has improved aggressive behavior in isolated mice $(94,95)$.

5. Herbal drugs: Central nervous system disease had been treated in ancient Korea and China until now, by Uwhangchungsimwon (UCW) as a herbal drug. Researchers have kept mice in separate cages to induce isolation for 31 days. Isolated mice were shown to be depressed, while those mice, which had oral administration of UCW every day, after 17 days, have shown improvement in behavioral tests and significantly reduced depressive-like behaviors. Improvements were justified according to an increased level of serum corticosterone and a higher level of dopamine, serotonin, and norepinephrine in the hippocampus. This investigation has shown that UCW consumption has diminished isolation-induced depression in mice by ameliorating neurochemicals (96).

\section{Non-drug treatments}

1. Environmental Enrichment (EE): It has been indicated alternations in neurotransmitters levels such as glutamate, serotonin, and decreased BDNF induced by chronic isolation were modified in EE (89). Social activity among rats exposed to a novel environment was higher than standard rats. These results proved the potentiality of nondrug protocols in improving mental deficits (97). Moreover, well-being in isolated patients is related to the quality of social support; consequently, any prescription to encourage being social and connected has been considered by the practitioner (98). EE, as a non-pharmacological treatment, has been applied in some investigation. Data have suggested that EE has enhanced social and cognitive deficits in isolated patients. Several researchers have exhibited the importance of positive and hopeful experiences in life to recover the brain from behavioral dysfunction (99).

Anti-depressant effects of EE have been demonstrated by investigations according to SI in rats (100). It has been shown that depression-related behavior and related abnormalities followed by long-term isolation, could be treated by EE. It can also perform as effective as fluoxetine; however, side effects associated with a pharmacological drug would not happen with EE treatment. Isolation-induced decrease in 5-HT level has been regulated through EE treatment, and the 5-HT level has increased in the hippocampus and prefrontal cortex (64). Finally, some investigations revealed that using an EE increases the rate of neurogenesis to maintain proliferation of dentate gyrus (DG) hippocampal cells in socially isolated mice, which results in maintaining social recognition memory and improving amnesic-like impairment (101).

In conclusion, EE plays a significant role in promoting neurogenesis in the hippocampus, impaired by social isolation. 
2. Exercise: Frequent physical exercise has been accepted among people to improve the physical and emotional conditions. By designing several studies on sports achievements, it has demonstrated that neurotransmitters' functionality and brain plasticity have been modulated in socially isolated rodents (102). In several types of research, rats were forced to run on a treadmill, which was a stressful condition. Results were disparate compared to voluntarily exercise, which resulted in regulating BDNF levels in isolated rats. Some other investigations have revealed that elective exercise cannot make any improvements in cognitive and social behavior impairments $(103,104)$. Eight weeks of running on a treadmill (30 min/day) have been shown to improve short-term and spatial working memory in SI rats (105). Regular treadmill exercise has improved isolation-induced depression-like behavior by regulating the hypothalamic-pituitary-adrenal (HPA) axis and this type of exercise has decreased stress hormones. Additionally, increased BDNF, NGF, serotonergic cells, and brain plasticity in the hippocampus have occurred after physical activity $(22,106)$.

Clinical research has revealed that walking among adults has positively affected social experiences for people who have been isolated and lived alone. At the end of this research, adults have claimed an improvement in their feelings and are encouraged to start social relationships, to get to know new people, and finally, to leave isolating mood (107). As stated by the research on rats, it has indicated that the development of monoaminergic axons has been prevented during isolation periods in maternal separation (108). Eventually, voluntarily running exercise has been found to stop emotional and social impairments by stimulating monoaminergic axons to start improvements again (109).

As claimed by more studies, pro-inflammatory and cytokine interleukin-1 $\beta$ (IL-1 $\beta$ ) has been produced increasingly in the hippocampus during the adolescence period. Besides, Social isolation adversely affects the hippocampus neurogenesis. Adolescence is a critical period in hippocampus maturation, and any detrimental impact makes more impairment in adulthood $(110,111)$. These Researches have found that aerobic exercises decreased stress induced by both adolescence and isolation in the hippocampus. Eventually, it has been demonstrated by some evidence that overexpression of IL-1 $\beta$ has been reversed by running and aerobic exercise during isolation housing in adolescence; while, supportive effects on neurogenesis occurred at the same time and resulted in developed recognition and social activity $(111,112)$. These findings explained how SI changed 5-hydroxytryptamine expression, and led to apoptosis in rats, which can account for cognition deficit and anxiety mood. Investigations have also designed an experiment to explore swimming effects on socially isolated old rats (113).

Tryptophan hydroxylase positive cell, 5-hydroxytryptamine positive cells, and Bcl-2 (B-cell lymphoma 2) expression have increased while BAX (Bcl-2-associated X protein) and cytochrome c expression were suppressed while swimming exercise. It was shown that swimming would lead to apoptosis prevention, reduced anxiety, and enhancement in social and learning capability in rats (114).

As claimed via an earlier investigation, depression was seen after periods of SI, which impaired the glutamatergic system in the hippocampus and NMDA receptor co-agonist D-serine. It has revealed that endurance exercise has attenuated adverse effects induced by isolation $(115,116)$. Amelioration of glutamate transmission has also decreased depressive behavior in rats. Therefore, exercising has been able to decline depression, social deficits, and cognition impairment induced by isolation experiences (117).

3. Music: Music is well known as a way to express emotion and has effects on well-being feeling, regulating hormones, and neurotransmitters. Physicians have found music therapy as a method to alleviate patients who had shown regression and weak sociability during housing isolation (118). Two types of patients, adults in the general ward of the hospital and children with leukemia in an isolated room, have experienced it as an enjoyable practice (119). They have claimed reduced fears, stress, and a motivated mood by listening to music. Besides, more verbalization, self-expression, and social relationships were reported. Beneficial impacts of listening to music have been performed by neurochemicals such as dopamine and oxytocin, which resulted in active talking and better communication (120). It has also enhanced health conditions in patients with Parkinson's disease by increasing social benefit.

In conclusion, music has been considered a non-pharmacological treatment in isolated patients and SI (121). 
4. Technology: As mentioned earlier, the environment itself plays a critical role in brain function and development. Lack of social interaction, as an absence of social stimulation on the brain, may lead to lesser cognitive reserve, lower brain flexibility, and cognitive impairment (122). There are several investigations on using smartphones, which have been used as a critical tool to connect humans and plays a crucial role in social capability and decreasing SI (123). Nowadays, social interaction has strictly wired into mobile phones via social platforms such as chat rooms, groups and channels, YouTube videos, and video-call applications like Skype. During the pandemic, when getting quarantine and staying at home was advised, social support has been provided mostly by social media throughout smartphones. People could join in social activities and feel like a helpful member of the community to reduce the detrimental effects of isolation (124). Social engagement has shown to improve age-related cognitive deficits, dementia, and memory decline induced by SI. Moreover, a sense of belonging and connection with others in places like school has shown to be crucial for academic success (125).

During the COVID-19 pandemic, Attitudes towards social robots have changed. Pieces of evidence have shown that people were encouraged to buy social robots more than ever. The emotional and behavioral features of those robots have made people feel less lonely and isolated; also, social support and reduced depressive-like mood were reported. A sense of happiness and having a better quality of life was seen through interactions with a robot, which was designed to behave socially interactive (126-128).

5. Farming: Farming activities have been shown to regulate social functioning for those who are suffering from a mental disorder. Several investigations have revealed that farming activities moderated getting into the community and having a connection with people. Being with each other is essential for people with mental problems; therefore, drug treatments will not work when social bonds are weak. According to the current research, social farming has been useful for social interaction and fihjting isolation $(129,130)$.

\section{Conclusion}

This review presented an overview of available studies on social isolation, adverse effects on cognition, and possible treatments. A wide range of studies have been collected and retrieved to explain beneficial treatment methods. Learning and memory impairments, anxiety and depressive-like behaviors, and social deficit consequences of social isolation have improved by prescription of some opioid drugs, anti-depressants, antipsychotics, and a variety of antagonists. Moreover, Antioxidants and Herbal medications have found helpful to ameliorate isolation side effects. Besides, an enriched environment, regular exercise, and music, as non-drug treatments, have shown to be beneficial for isolated people. Finally, the application of technology and farming activities have suggested improving isolation-induced cognitive and social impairments.

\section{Authors' contributions}

All authors were involved in the conception and writing of manuscript.

\section{Declarations of interest}

This study was supported by Kerman Neuroscience Research Center, Kerman University of Medical Sciences, Kerman, Iran.

Since the review articles analyzes or discusses research previously published by others, rather than reporting new experimental result therefore, no consent has been used in this study.

\section{References}

1 Kotsou I, Quoidbach J, Hansenne M, Weytens F, Dupuis P, Mikolajczak MJE. Increasing emotional competence improves psychological and physical well-being, social relationships, and employability. 2011;11(2):354.

2. National Academies of Sciences E, Medicine. Social Isolation and Loneliness in Older Adults: Opportunities for the Health Care System. 2020.

3. Hays K, Shepard Payne JJJoPC, Counseling. Lived Experience, Transparency, Help, and Humility: Four Characteristics of Clergy Responding to Mental and Emotional Problems. 2020;74(1):4-11. 
4. Leigh-Hunt N, Bagguley D, Bash K, Turner V, Turnbull S, Valtorta N, et al. An overview of systematic reviews on the public health consequences of social isolation and loneliness. 2017;152:157-71.

5. de Jong Gierveld J, Van Tilburg T, Dykstra PAJChopr. Loneliness and social isolation. 2006:485-500.

6. Calati R, Ferrari C, Brittner M, Oasi O, Olié E, Carvalho AF, et al. Suicidal thoughts and behaviors and social isolation: A narrative review of the literature. 2019;245:653-67.

7. Cattan M, White M, Bond J, Learmouth AJA, society. Preventing social isolation and loneliness among older people: a systematic review of health promotion interventions. 2002.

8. Marziali ME, Card KG, McLinden T, Wang L, Trigg J, Hogg RSJA, et al. Physical distancing in COVID-19 may exacerbate experiences of social isolation among people living with HIV. 2020:1.

9. Banerjee D, Rai M. Social isolation in Covid-19: The impact of loneliness. SAGE Publications Sage UK: London, England; 2020.

10. Cacioppo JT, Hawkley LCJTics. Perceived social isolation and cognition. 2009;13(10):447-54.

11. Yang R, Wang H, Edelman LS, Tracy EL, Demiris G, Sward KA, et al. Loneliness as a mediator of the impact of social isolation on cognitive functioning of Chinese older adults. 2020.

12. Campagne DMJAog, geriatrics. Stress and perceived social isolation (loneliness). 2019;82:192-9.

13. Lin Y-S, Lin Y-F, Chen KC, Yang YK, Hsiao Y-HJN. Collapsin response mediator protein 5 (CRMP5) causes social deficits and accelerates memory loss in an animal model of Alzheimer's disease. 2019;157:107673.

14. Knox SS, Uvnäs-Moberg KJP. Social isolation and cardiovascular disease: an atherosclerotic pathway? $1998 ; 23(8): 877-90$.

15. Matthews T, Danese A, Wertz J, Odgers CL, Ambler A, Moffitt TE, et al. Social isolation, loneliness and depression in young adulthood: a behavioural genetic analysis. 2016;51(3):339-48.

16. Sanders CE, Field TM, Diego M, Kaplan MJA. The relationship of Internet use to depression and social isolation among adolescents. 2000;35(138):237-.

18. Santini ZI, Jose PE, Cornwell EY, Koyanagi A, Nielsen L, Hinrichsen C, et al. Social disconnectedness, perceived isolation, and symptoms of depression and anxiety among older Americans (NSHAP): a longitudinal mediation analysis. 2020;5(1):e62-e70.

18. Shankar A, McMunn A, Banks J, Steptoe AJHP. Loneliness, social isolation, and behavioral and biological health indicators in older adults. 2011;30(4):377.

19. Smith KJN, Reviews B. The association between loneliness, social isolation and inflammation: A systematic review and meta-analysis. 2020.

20. Begni V, Sanson A, Pfeiffer N, Brandwein C, Inta D, Talbot SR, et al. Social isolation in rats: Effects on animal welfare and molecular markers for neuroplasticity. 2020;15(10):e0240439.

21. Ieraci A, Mallei A, Popoli MJNp. Social isolation stress induces anxious-depressive-like behavior and alterations of neuroplasticity-related genes in adult male mice. 2016;2016.

22. Park H-S, Kim T-W, Park S-S, Lee S-JJJoER. Swimming exercise ameliorates mood disorder and memory impairment by enhancing neurogenesis, serotonin expression, and inhibiting apoptosis in social isolation rats during adolescence. 2020;16(2):132.

23. Helmich RC, Bloem BRJJoPsd. The impact of the COVID-19 pandemic on Parkinson's disease: hidden sorrows and emerging opportunities. 2020;10(2):351.

24. Feuillet L, Reuter F, Audoin B, Malikova I, Barrau K, Cherif AA, et al. Early cognitive impairment in patients with clinically isolated syndrome suggestive of multiple sclerosis. 2007;13(1):124-7. 
25. Wallace DL, Han M-H, Graham DL, Green TA, Vialou V, Iniguez SD, et al. CREB regulation of nucleus accumbens excitability mediates social isolation-induced behavioral deficits. 2009;12(2):200.

26. Mohammed A, El-Bakly WM, Ali A, El-Demerdash EJBBR. Rosuvastatin improves olanzapine's effects on behavioral impairment and hippocampal, hepatic and metabolic damages in isolated reared male rats. 2020;378:112305.

27. Read S, Comas-Herrera A, Grundy EJTJoGSB. Social Isolation and memory decline in later-life. 2020;75(2):367-76.

28. Wang B, Wu Q, Lei L, Sun H, Michael N, Zhang X, et al. Long-term social isolation inhibits autophagy activation, induces postsynaptic dysfunctions and impairs spatial memory. 2019;311:213-24.

29. Evans IE, Llewellyn DJ, Matthews FE, Woods RT, Brayne C, Clare L, et al. Social isolation, cognitive reserve, and cognition in healthy older people. 2018;13(8):e0201008.

30. Zorzo C, Méndez-López M, Méndez M, Arias JLJBbr. Adult social isolation leads to anxiety and spatial memory impairment: Brain activity pattern of COx and c-Fos. 2019;365:170-7.

31. Teo AR, Lerrigo R, Rogers MAJJoAD. The role of social isolation in social anxiety disorder: A systematic review and meta-analysis. 2013;27(4):353-64.

32. Robb CE, de Jager CA, Ahmadi-Abhari S, Giannakopoulou P, Udeh-Momoh C, McKeand J, et al. Associations of social isolation with anxiety and depression during the early COVID-19 pandemic: a survey of older adults in London, UK. 2020;11.

33. Milman E, Lee SA, Neimeyer RAJDS. Social isolation and the mitigation of coronavirus anxiety: The mediating role of meaning. 2020:1-13.

34. Li M, Wang W, Sun L, Du W, Zhou H, Shao FJBp. Chronic clozapine treatment improves the alterations of prepulse inhibition and BDNF mRNA expression in the medial prefrontal cortex that are induced by adolescent social isolation. 2019;30(4):311-9.

35. Kojima M, Otabi H, Kumanogoh H, Toyoda A, Ikawa M, Okabe M, et al. Reduction in BDNF from Inefficient Precursor Conversion Influences Nest Building and Promotes Depressive-Like Behavior in Mice. 2020;21(11):3984.

36. Tomasello MJJftToSB. The role of roles in uniquely human cognition and sociality. 2020;50(1):2-19.

37. Pietropaolo S, Sun Y, Li R, Brana C, Feldon J, Yee BKJBn. Limited impact of social isolation on Alzheimer-like symptoms in a triple transgenic mouse model. 2009;123(1):181.

38. Diwakarla S, Finkelstein DI, Constable R, Artaiz O, Di Natale M, McQuade RM, et al. Chronic isolation stress is associated with increased colonic and motor symptoms in the A53T mouse model of Parkinson's disease. 2020;32(3):e13755.

39. Victorino DB, Guimarães-Marques M, Nejm M, Scorza FA, Scorza CAJJoPsd. COVID-19 and Parkinson's Disease: Are We Dealing with Short-term Impacts or Something Worse? 2020;10(3):899.

40. Chu J, Schulz LEJARoDP. Play, curiosity, and cognition. 2020;2.

41. Niesink RJ, Van Ree JMJN. Involvement of opioid and dopaminergic systems in isolation-induced pinning and social grooming of young rats. 1989;28(4):411-8.

42. Zhao C, Chang L, Auger AP, Gammie SC, Riters LVJG, Brain, Behavior. Mu opioid receptors in the medial preoptic area govern social play behavior in adolescent male rats. 2020.

43. Goodwin GA, Molina VA, Spear LPJDPTJotISfDP. Repeated exposure of rat pups to isolation attenuates isolation-induced ultrasonic vocalization rates: Reversal with naltrexone. 1994;27(1):53-64. 
44. Lewis MH, Gluck JP, Beauchamp AJ, Keresztury MF, Mailman RBJBR. Long-term effects of early social isolation in Macaca mulatta: changes in dopamine receptor function following apomorphine challenge. 1990;513(1):67-73.

45. Hashizume T, Haglof S, Malven PJJoas. Intracerebral methionine-enkephalin, serum cortisol, and serum $\beta$-endorphin during acute exposure of sheep to physical or isolation stress. 1994;72(3):700-8.

46. Zheng S, Masuda T, Matsunaga M, Noguchi Y, Ohtsubo Y, Yamasue H, et al. Oxytocin Receptor Gene (OXTR) and Childhood Adversity Influence Trust. 2020;121:104840.

47. Grippo AJ, Trahanas DM, Zimmerman II RR, Porges SW, Carter CSJP. Oxytocin protects against negative behavioral and autonomic consequences of long-term social isolation. 2009;34(10):1542-53.

48. Kramer KM, Cushing BS, Carter CSJP, behavior. Developmental effects of oxytocin on stress response: single versus repeated exposure. 2003;79(4-5):775-82.

49. Wagner B, Relling A, Kieffer J, Parker AJDae. Intranasal oxytocin treatment does not attenuate the hypothalamo-pituitary-adrenal axis in beef heifers subjected to isolation stress or restraint and isolation stress. 2020;70:106379.

50. Panksepp J. Oxytocin effects on emotional processes: separation distress, social bonding, and relationships to psychiatric disorders. 1992.

51. Kenkel W, Perkeybile A-M, Yee J, Pournajafi-Nazarloo H, Lillard T, Ferguson E, et al. Behavioral and epigenetic consequences of oxytocin treatment at birth. 2019;5(5):eaav2244.

52. Ebstein RP, Israel S, Lerer E, Uzefovsky F, Shalev I, Gritsenko I, et al. Arginine vasopressin and oxytocin modulate human social behavior. 2009.

53. Baracz SJ, Everett NA, Robinson KJ, Campbell GR, Cornish JLJJoN. Maternal separation changes maternal care, anxiety-like behaviour and expression of paraventricular oxytocin and corticotrophin-releasing factor immunoreactivity in lactating rats. 2020;32(6):e12861.

54. Jirikowski GFJC, research t. Diversity of central oxytocinergic projections. 2019;375(1):41-8.

55. Han RT, Kim Y-B, Park E-H, Kim JY, Ryu C, Kim HY, et al. Long-term isolation elicits depression and anxiety-related behaviors by reducing oxytocin-induced GABAergic transmission in central amygdala. 2018;11:246.

56. Stevenson JR, McMahon EK, Boner W, Haussmann MFJP. Oxytocin administration prevents cellular aging caused by social isolation. 2019;103:52-60.

57. Allaman I, Fiumelli H, Magistretti PJ, Martin J-LJP. Fluoxetine regulates the expression of neurotrophic/growth factors and glucose metabolism in astrocytes. 2011;216(1):75-84.

58. Larsen MH, Mikkelsen JD, Hay-Schmidt A, Sandi CJJopr. Regulation of brain-derived neurotrophic factor (BDNF) in the chronic unpredictable stress rat model and the effects of chronic antidepressant treatment. 2010;44(13):808-16.

59. Nin MS, Martinez L, Pibiri F, Nelson M, Pinna GJFie. Neurosteroids reduce social isolation-induced behavioral deficits: a proposed link with neurosteroid-mediated upregulation of BDNF expression. 2011;2:73.

60. Dong E, Matsumoto K, Uzunova V, Sugaya I, Takahata H, Nomura H, et al. Brain $5 \alpha-$ dihydroprogesterone and allopregnanolone synthesis in a mouse model of protracted social isolation. 2001;98(5):2849-54.

61. Pinna G, Costa E, Guidotti AJP. Fluoxetine and norfluoxetine stereospecifically and selectively increase brain neurosteroid content at doses that are inactive on 5-HT reuptake. 2006;186(3):362-72. 
62. Perić I, Costina V, Stanisavljević A, Findeisen P, Filipović DJN. Proteomic characterization of hippocampus of chronically socially isolated rats treated with fluoxetine: Depression-like behaviour and fluoxetine mechanism of action. 2018;135:268-83.

63. Ibi D, Takuma K, Koike H, Mizoguchi H, Tsuritani K, Kuwahara Y, et al. Social isolation rearinginduced impairment of the hippocampal neurogenesis is associated with deficits in spatial memory and emotion-related behaviors in juvenile mice. 2008;105(3):921-32.

64. Guarnieri LO, Pereira-Caixeta AR, Medeiros DC, Aquino NS, Szawka RE, Mendes EM, et al. Proneurogenic effect of fluoxetine in the olfactory bulb is concomitant to improvements in social memory and depressive-like behavior of socially isolated mice. 2020;10(1):1-14.

65. Brenes JC, Fornaguera JJBbr. The effect of chronic fluoxetine on social isolation-induced changes on sucrose consumption, immobility behavior, and on serotonin and dopamine function in hippocampus and ventral striatum. 2009;198(1):199-205.

66. Möller M, Du Preez JL, Viljoen FP, Berk M, Emsley R, Harvey BHJB, behavior,, et al. Social isolation rearing induces mitochondrial, immunological, neurochemical and behavioural deficits in rats, and is reversed by clozapine or N-acetyl cysteine. 2013;30:156-67.

67. Contreras-Shannon V, Heart DL, Paredes RM, Navaira E, Catano G, Maffi SK, et al. Clozapine-induced mitochondria alterations and inflammation in brain and insulin-responsive cells. 2013;8(3):e59012.

68. Filipović D, Stanisavljević A, Jasnić N, Bernardi RE, Inta D, Perić I, et al. Chronic treatment with fluoxetine or clozapine of socially isolated rats prevents subsector-specific reduction of parvalbumin immunoreactive cells in the hippocampus. 2018;371:384-94.

69. Manzaneque JM, Brain PF, Navarro JFJPiN-p, Psychiatry B. Effect of low doses of clozapine on behaviour of isolated and group-housed male mice in the elevated plus-maze test. 2002;26(2):349-55.

70. Todorović N, Filipović DJPB, Behavior. The antidepressant-and anxiolytic-like effects of fluoxetine and clozapine in chronically isolated rats involve inhibition of hippocampal TNF- $\alpha$. 2017;163:57-65.

71. Todorović N, Mićić B, Schwirtlich M, Stevanović M, Filipović DJN. Subregion-specific protective effects of fluoxetine and clozapine on parvalbumin expression in medial prefrontal cortex of chronically isolated rats. 2019;396:24-35.

72. Pourahmad J, Salimi A, Imani F, Jamali Z, Ahvar NJIPA. The Clozapine-induced Toxicity via Induction of Oxidative Stress and Mitochondrial Dysfunction in Human Blood Lymphocytes and Protecting role of L-Carnitine. 2020;3(1):3e9: 1-9.

73. Stanisavljević A, Perić I, Bernardi RE, Gass P, Filipović DJBrb. Clozapine increased c-fos protein expression in several brain subregions of socially isolated rats. 2019;152:35-44.

74. Li N, Wu X, Li LJBp. Chronic administration of clozapine alleviates reversal-learning impairment in isolation-reared rats. 2007;18(2):135-45.

75. McLean SL. Exploration of cognitive and neurochemical deficits in an animal model of schizophrenia. Investigation into sub-chronic PCP-induced cognitive deficits using behavioural, neurochemical and electrophysiological techniques; and use of receptor-selective agents to study the pharmacology of antipsychotics in female rats: University of Bradford; 2010.

76. Hagan JJ, Jones DNJSB. Predicting drug efficacy for cognitive deficits in schizophrenia. 2005;31(4):83053.

77. Alvarez RJ, Pafundo DE, Zold CL, Belforte JEJJoN. Interneuron NMDA receptor ablation induces hippocampus-prefrontal cortex functional hypoconnectivity after adolescence in a mouse model of schizophrenia. 2020;40(16):3304-17. 
78. Ouchi H, Ono K, Murakami Y, Matsumoto KJBbr. Social isolation induces deficit of latent learning performance in mice: a putative animal model of attention deficit/hyperactivity disorder. 2013;238:146-53.

79. Scaccianoce S, Del Bianco P, Paolone G, Caprioli D, Modafferi AM, Nencini P, et al. Social isolation selectively reduces hippocampal brain-derived neurotrophic factor without altering plasma corticosterone. $2006 ; 168(2): 323-5$.

80. Piazza PV, Le Moal MJBr. Social isolation-induced enhancement of the psychomotor effects of morphine depends on corticosterone secretion. 1994;640(1-2):136-9.

81. Garcia-Mompo C, Curto Y, Carceller H, Gilabert-Juan J, Rodriguez-Flores E, Guirado R, et al. $\Delta$-9Tetrahydrocannabinol treatment during adolescence and alterations in the inhibitory networks of the adult prefrontal cortex in mice subjected to perinatal NMDA receptor antagonist injection and to postweaning social isolation. 2020;10(1):1-13.

82. Marsden CA, King MV, Fone KCJN. Influence of social isolation in the rat on serotonergic function and memory-relevance to models of schizophrenia and the role of 5-HT6 receptors. 2011;61(3):400-7.

83. King MV, Seeman P, Marsden CA, Fone KCJS. Increased dopamine D receptors in rats reared in social isolation. 2009;63(6):476-83.

84. Porkess MV. The impact of social isolation on rat behaviour: University of Nottingham; 2008.

85. Watson DJ, Marsden CA, Millan MJ, Fone KCJIJoN. Blockade of dopamine D3 but not D2 receptors reverses the novel object discrimination impairment produced by post-weaning social isolation: implications for schizophrenia and its treatment. 2012;15(4):471-84.

86. Lv L, Liu Y, Xie J, Wu Y, Zhao J, Li Q, et al. Interplay between $\alpha 2$-chimaerin and Rac1 activity determines dynamic maintenance of long-term memory. 2019;10(1):1-12.

87. Zhang X-Q, Jiang H-J, Xu L, Yang S-Y, Wang G-Z, Jiang H-D, et al. The metabotropic glutamate receptor 2/3 antagonist LY341495 improves working memory in adult mice following juvenile social isolation. 2020;177:108231.

88. Sweeten BL, Adkins AM, Wellman LL, Sanford LDJPiN-P, Psychiatry B. Group II metabotropic glutamate receptor activation in the basolateral amygdala mediates individual differences in stress-induced changes in rapid eye movement sleep. 2020:110014.

89. Niu L, Luo SS, Xu Y, Wang Z, Luo D, Yang H, et al. The critical role of the hippocampal NLRP3 inflammasome in social isolation-induced cognitive impairment in male mice. 2020;175:107301.

90. Garcia EJ, Cain MEJPB, Behavior. Environmental enrichment and a selective metabotropic glutamate receptor2/3 (mGluR2/3) agonist suppress amphetamine self-administration: Characterizing baseline differences. 2020:172907.

91. Wang Q, Liu Y, Zhang J, Wang WJFiP. Corticotropin-Releasing Factor Receptors in the Locus Coeruleus Modulate the Enhancement of Active Coping Behaviors Induced by Chronic Predator Odor Inoculation in Mice. 2020;10:3028.

92. Wagner SJC, research t. Urocortins and their unfolding role in mammalian social behavior. 2019;375(1):133-42.

93. Atmore KH, Stein DJ, Harvey BH, Russell VA, Howells FMJEN. Differential effects of social isolation rearing on glutamate-and GABA-stimulated noradrenaline release in the rat prefrontal cortex and hippocampus. 2020;36:111-20.

94. Hira S, Saleem U, Anwar F, Ahmad BJFiP. Antioxidants attenuate isolation-and L-DOPA-induced aggression in mice. 2018;8:945. 
95. Vekiari S, Tzia C, Oreopoulou V, Thomopoulos CJRIDSG. Isolation of natural antioxidants from oregano. 1993.

96. Oh H-M, Lee J-S, Kim S-W, Oh Y-T, Kim W-Y, Lee S-B, et al. Uwhangchungsimwon, a standardized herbal drug, exerts an anti-depressive effect in a social isolation stress-induced mouse model. 2020;10:1674.

97. Bespalov AY, van Gaalen MM, Gross G. Antidepressant treatment in anxiety disorders. Behavioral Neurobiology of Anxiety and Its Treatment: Springer; 2009. p. 361-90.

98. Rook KS. The functions of social bonds: Perspectives from research on social support, loneliness and social isolation. Social support: Theory, research and applications: Springer; 1985. p. 243-67.

99. Grippo AJ, Ihm E, Wardwell J, McNeal N, Scotti M-AL, Moenk DA, et al. The effects of environmental enrichment on depressive-and anxiety-relevant behaviors in socially isolated prairie voles. 2014;76(4):277.

100. Cao M, Pu T, Wang L, Marshall C, He H, Hu G, et al. Early enriched physical environment reverses impairments of the hippocampus, but not medial prefrontal cortex, of socially-isolated mice. 2017;64:232-43.

101. Segovia G, Yagüe AG, García-Verdugo JM, Mora FJBrb. Environmental enrichment promotes neurogenesis and changes the extracellular concentrations of glutamate and GABA in the hippocampus of aged rats. 2006;70(1):8-14.

102. Brenes JC, Fornaguera J, Sequeira-Cordero AJFiP. Environmental Enrichment and Physical Exercise Attenuate the Depressive-Like Effects Induced by Social Isolation Stress in Rats. 2020;11:804.

103. Hong Y-P, Lee H-C, Kim H-TJJoen, biochemistry. Treadmill exercise after social isolation increases the levels of NGF, BDNF, and synapsin I to induce survival of neurons in the hippocampus, and improves depression-like behavior. 2015;19(1):11.

104. Cho J-W, Jung S-Y, Lee S-W, Lee S-J, Seo T-B, Kim Y-P, et al. Treadmill exercise ameliorates social isolation-induced depression through neuronal generation in rat pups. 2017;13(6):627.

105. Cechetti F, Fochesatto C, Scopel D, Nardin P, Gonçalves CA, Netto CA, et al. Effect of a neuroprotective exercise protocol on oxidative state and BDNF levels in the rat hippocampus. 2008;1188:182-8.

106. Tai F, Wang C, Deng X, Li R, Guo Z, Quan H, et al. Treadmill exercise ameliorates chronic REM sleep deprivation-induced anxiety-like behavior and cognitive impairment in C57BL/6J mice. 2020;164:198-207.

107. Travis SS, Duncan HH, McAuley WJJJoPN, Services MH. Mall walking: An effective mental health intervention for older adults. 1996;34(8):36-8.

108. Hwang J, Wang L, Siever J, Medico TD, Jones CAJA, health m. Loneliness and social isolation among older adults in a community exercise program: a qualitative study. 2019;23(6):736-42.

109. Okudan N, Belviranlı MJN. Long-term voluntary exercise prevents post-weaning social isolation-induced cognitive impairment in rats. 2017;360:1-8.

110. Raz N, Daugherty AM, Bender AR, Dahle CL, Land SJBS, Function. Volume of the hippocampal subfields in healthy adults: differential associations with age and a pro-inflammatory genetic variant. 2015;220(5):2663-74.

111. Wagner G, Herbsleb M, Cruz Fdl, Schumann A, Brünner F, Schachtzabel C, et al. Hippocampal structure, metabolism, and inflammatory response after a 6-week intense aerobic exercise in healthy young adults: a controlled trial. 2015;35(10):1570-8.

112. Jazaieri H, Goldin PR, Werner K, Ziv M, Gross JJJJocp. A randomized trial of MBSR versus aerobic exercise for social anxiety disorder. 2012;68(7):715-31.

113. Heritch AJ, Henderson K, Westfall TCJJopr. Effects of social isolation on brain catecholamines and forced swimming in rats: prevention by antidepressant treatment. 1990;24(3):251-8. 
114. Park S-S, Park H-S, Kim T-W, Lee S-JJJoER. Effects of swimming exercise on social isolation-induced memory impairment and apoptosis in old rats. 2020;16(3):234.

115. Gómez-Galán M, Femenía T, Åberg E, Graae L, Van Eeckhaut A, Smolders I, et al. Running opposes the effects of social isolation on synaptic plasticity and transmission in a rat model of depression. 2016;11(10):e0165071.

116. Jang YJN. Endurance exercise-induced expression of autophagy-related protein coincides with anabolic expression and neurogenesis in the hippocampus of the mouse brain. 2020;31(6):442-9.

117. Imboden C, Gerber M, Beck J, Holsboer-Trachsler E, Pühse U, Hatzinger M. Aerobic exercise or stretching as add-on to inpatient treatment of depression: Similar antidepressant effects on depressive symptoms and larger effects on working memory for aerobic exercise alone. Journal of Affective Disorders. 2020;276:86676 .

118. Robb SL. The Effect of Therapeutic Music Interventions on the Behavior of Hospitalized Children in Isolation: Developing a Contextual Support Model of Music Therapy. Journal of Music Therapy. 2000;37(2):11846.

119. Brodsky W. Music Therapy as an Intervention for Children with Cancer in Isolation Rooms1. Music Therapy. 1989;8(1):17-34.

120. Zoltán J. Musica et medicina. Orvosi Hetilap OH. 2019;160(11):403-18.

121. Haneishi EJJomt. Effects of a music therapy voice protocol on speech intelligibility, vocal acoustic measures, and mood of individuals with Parkinson's disease. 2001;38(4):273-90.

122. Garcia-Constantino M, Synnott J, Nugent C, Burns A, Little M, Samson D, editors. The desirability of the use of technology to prevent social isolation of older adults. Proceedings of the 32nd International BCS Human Computer Interaction Conference 32; 2018.

123. Cho JJC, behavior, networking s. Roles of smartphone app use in improving social capital and reducing social isolation. 2015;18(6):350-5.

124. Chen Y-RR, Schulz PJJJomIr. The effect of information communication technology interventions on reducing social isolation in the elderly: a systematic review. 2016;18(1):e18.

125. Zamir S, Hennessy CH, Taylor AH, Jones RBJBg. Video-calls to reduce loneliness and social isolation within care environments for older people: an implementation study using collaborative action research. 2018;18(1):62.

126. Ghafurian M, Ellard C, Dautenhahn KJapa. Social Companion Robots to Reduce Isolation: A Perception Change Due to COVID-19. 2020.

127. Henkel AP, Čaić M, Blaurock M, Okan MJJoSM. Robotic transformative service research: deploying social robots for consumer well-being during Covid-19 and beyond. 2020.

128. Hwang T-J, Rabheru K, Peisah C, Reichman W, Ikeda MJIP. Loneliness and Social Isolation during the COVID-19 Pandemic. 2020:1-15.

129. De Vivo C, Ascani M, Gaito MJIRoAE. Social Farming and inclusion in EU ESI Funds programming. 2019;74(2):53-60.

130. Moruzzo R, Di Iacovo F, Funghi A, Scarpellini P, Diaz SE, Riccioli FJSS. Social farming: An inclusive environment conducive to participant personal growth. 2019;8(11):301. 